\title{
Sequential treatment with doxorubicin and zoledronic acid has no additive effects in an aggressive model of established bone metastases
} \author{
Käkönen ${ }^{2,4}$ \\ 'Pharmatest Services Ltd., Turku Fl-20520, Finland. \\ ${ }^{2}$ Aurexel Life Sciences Ltd., Askainen FI-21240, Finland. \\ ${ }^{3}$ PreclinApps Ltd., Raisio FI-21200, Finland. \\ ${ }^{4}$ Institute of Biomedicine, University of Turku, Turku 20520, Finland.
}

Mari I. Suominen'1, Rami Käkönen², Jukka P. Rissanen ${ }^{3}$, Jussi M. Halleen', Pirkko Härkönen ${ }^{4}$, Sanna-Maria

Correspondence to: Dr. Mari I. Suominen, Pharmatest Services Ltd., Itäinen Pitkäkatu 4C, Turku, FI-20520, Finland.

E-mail: mari.suominen@pharmatest.com

How to cite this article: Suominen MI, Käkönen R, Rissanen JP, Halleen JM, Härkönen P, Käkönen SM. Sequential treatment with doxorubicin and zoledronic acid has no additive effects in an aggressive model of established bone metastases. $J$ Cancer Metastasis Treat 2019;5:14. http://dx.doi.org/10.20517/2394-4722.2018.64

Received: 29 Sep 2018 First Decision: 16 Nov 2018 Revised: 3 Jan 2019 Accepted: 14 Jan 2019 Published: 28 Feb 2019

Science Editor: Andrea Nicolini Copy Editor: Cui Yu Production Editor: Huan-Liang Wu

\begin{abstract}
Aim: Bisphosphonates are used as an adjuvant treatment in breast cancer bone metastasis patients, often simultaneously with chemotherapeutic agents. Interestingly, their sequential combination has been reported to have synergistic anti-tumor effects on bone metastases in preclinical models. We studied the effects of doxorubicin (DOX) and zoledronic acid (ZOL) and their combination on established bone metastases in the MDA-MB-231(SA) GFP bone metastasis model.
\end{abstract}

Methods: Tumor burden and osteolytic bone lesions were quantitated by fluorescence imaging and radiography, respectively. The mice were randomized in four groups receiving vehicle, DOX, ZOL or both DOX and ZOL in a sequential combination on day 14 . Serum marker of osteoclast number was followed weekly, and blood ionized calcium was measured at sacrifice. Bone and tumor area, apoptosis and proliferation of tumor cells were analyzed from histological sections.

Results: ZOL prevented hypercalcemia and osteolytic lesion progression, whereas DOX induced apoptosis in the MDA-MB-231(SA)GFP cells. However, neither of the treatments alone nor in sequential combination were able to reduce tumor burden in bone. Furthermore, no additive effects on tumor cell apoptosis were observed in the

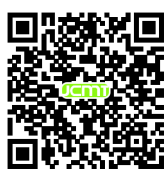


combination group.

Conclusion: No additive effects in combination of DOX and ZOL were observed in this aggressive model of breast cancer bone metastasis.

Keywords: Combination treatment, breast cancer, bone metastasis, MDA-MB-231(SA), doxorubicin, zoledronic acid

\section{INTRODUCTION}

Despite major advances in the treatment of primary breast cancer within the past decades, treatment of advanced breast cancer with bone metastases is still palliative in nature, as no curative treatments are available. Inhibitors of bone resorption, such as zoledronic acid (ZOL) or denosumab are used as adjuvant treatment in breast cancer patients with bone metastases, often simultaneously with chemotherapeutic agents such as doxorubicin ${ }^{[1,2]}$. In these patients bisphosphonates decrease bone pain and skeletal-related events, but do not increase the overall survival ${ }^{[3]}$. However, before the formation of bone metastases, bisphosphonate treatment in combination with standard chemotherapy may prevent the occurrence of bone metastases in postmenopausal, early stage breast cancer patients ${ }^{[4]}$. Many preclinical studies in established experimental breast cancer bone metastasis models have reported direct anti-tumor effects of bisphosphonates alone, such as increase of apoptosis in intraosseous tumors and improved survival ${ }^{[5-7]}$, but we have reported no effects on survival in a model of established bone metastases ${ }^{[8]}$.

Bisphosphonates seek and incorporate into the hydroxyapatite in bone matrix. Therefore, it has remained an unanswered question how tumor cells in soft tissues can be exposed to effective concentrations of bisphosphonates. It is also possible that what was believed to be a direct anti-tumor effect is indeed, indirect. It has been shown that macrophages take up bisphosphonates in cytotoxic quantities, and tumor growth in soft tissues can be inhibited due to the lack of tumor-associated macrophages ${ }^{[9,10]}$.

Some exploratory preclinical studies investigating the effects of combinations of aminobisphosphonates and chemotherapeutic agents have suggested additional benefits. Sequential treatment with doxorubicin (DOX) followed by a single dose of ZOL $24 \mathrm{~h}$ later was reported to decrease tumor volume and proliferation and to increase apoptosis as well as mouse survival with established bone metastases with MDA-MB231/BO2 breast cancer cells ${ }^{[11]}$. In addition, a single cycle of sequential administration of DOX and ZOL in a very early phase, 2 days after cancer cell inoculation, was reported to decrease intraosseus tumor area in an intracardiac mouse model using MDA-MB231 cells with green fluorescent protein (GFP) ${ }^{[12]}$. Three additional studies by the same group with MDA-MB436 breast cancer cells showed synergistic effects on tumor volume reduction with the weekly administration of DOX and ZOL in combination, both on bone metastasis and primary tumor $^{[13-15]}$. In these studies, the combination treatment reduced the intraosseous tumor area and increased survival compared to DOX or ZOL monotherapy. The mode-of-action of the combination is not understood, but increased uptake of ZOL by tumor cells was observed with the sequential combination ${ }^{[14]}$. Because of the varying results of anti-tumor effects of bisphosphonates, we felt a need to investigate these effects in a fairly aggressive and widely used model of breast cancer bone metastases, utilizing the human MDA-MB231(SA) GFP cells in nude mice. The treatment schedule was otherwise the same as used by Ottewell et al ${ }^{[13]}$, but the treatment was started 3 days earlier to accommodate the more aggressive model.

\section{METHODS}

\section{Compounds and cell line}

DOX (Sigma-Aldrich, Saint Louis, MO, USA) was dissolved in saline (0.9\% NaCl, Baxter, Deerfield, IL, USA) to concentration of $0.5 \mathrm{mg} / \mathrm{mL}$. ZOL (Zometa', Novartis Pharma GmbH, Basel, Switzerland) infusion 
concentrate $(0.8 \mathrm{mg} / \mathrm{mL})$ was diluted in saline to obtain the concentration of $0.02 \mathrm{mg} / \mathrm{mL}$. Saline was used as a vehicle control. MDA-MB231(SA)GFP ${ }^{[8]}$ were authenticated using short tandem repeat analysis (GenePrint10 system, Promega, Madison, WI, USA) at Institute for Molecular Medicine Finland (FIMM, Helsinki, Finland).

\section{In vivo study}

The animal experiment was approved by the Animal Experiment Board of Finland and performed according to European Union directive 2010/63/EU. Five-week-old female athymic nude mice (Hsd: Athymic nudenu, Harlan Laboratories B.V./Envigo, Horst, The Netherlands) were used. The mice were housed in groups of five in Scantainer ${ }^{\text {mi }}$ (Scanbur, Karlslunde, Denmark) and fed an autofluorescence-reducing chlorophyll-free purified diet (AIN-76A, Special Diets Services, Witham, UK). The animals were weighed three times per week and daily during the last five days. On day $0,10^{5} \mathrm{MDA}-\mathrm{MB} 231$ (SA)GFP breast cancer cells were inoculated into the left cardiac ventricle of the animals, as described previously ${ }^{[16,17]}$. The mice were anesthetized with 4 mg/kg xylazine (Rompun Vet 20 mg/mL, Bayer Animal Health GmbH, Leverkusen, Germany) and 75 mg/kg ketamine (Ketalar $10 \mathrm{mg} / \mathrm{mL}$, Pfizer, NY, USA) intramuscularly (im) for inoculations and imaging procedures. Buprenorphine (Temgesic, Invidior UK Ltd, Slough, UK) was used as analgesic at $0.1 \mathrm{mg} / \mathrm{kg}$ subcutaneously (s.c.) and was administered once before the intracardiac inoculations and twice a day during the last five days of the study. The mice were randomized into four treatment groups $(n=8)$ on day 14 based on tumor growth and osteolytic lesion size as well as body weight. Tumor growth and osteolytic lesion size were measured by fluorescence imaging and X-ray analysis, respectively, on study day 14 and at sacrifice. The mice were sacrificed on day 25, except the 2-3 mice per group, which were sacrificed earlier due to weight loss over $20 \%$ or paraplegia. All the mice were included in the endpoint analyses. The mean day of sacrifice was very similar in all groups ranging from 24.13 in the DOX group to 24.63 in the ZOL group.

The control group received vehicle $5 \mathrm{~mL} / \mathrm{kg}$ s.c. on day 15 and intraperitoneally (i.p.) on days 15 and 22. The DOX group received half of the maximal tolerated dose, DOX $2.5 \mathrm{mg} / \mathrm{kg}$ i.p. on days 15 and 22 . The ZOL group received a single dose of ZOL $0.1 \mathrm{mg} / \mathrm{kg}$ s.c. on day 15 . The DOX + ZOL group received DOX $(2.5 \mathrm{mg} / \mathrm{kg}$ i.p.) on days 15 and 22 and a single dose of ZOL (0.1 mg/kg s.c.) $24 \mathrm{~h}$ after the first dose of DOX, i.e, on day 16 (schedule of the combination group dosing is shown in Figure 1).

Blood samples were collected from saphenous vein to $100 \mu \mathrm{L}$ Microvette serum tubes (Sarstedt AG\&Co, Nümbrecht, Germany), on days 1, 9, 17 and 24. Terminal blood samples were obtained by cardiac puncture. Five intact animals of the same strain and age were sacrificed on day 25 in order to obtain the baseline level for blood $\mathrm{Ca}^{2+}$ (ionized calcium). Femur and tibia were collected for histomorphometric analyses.

\section{Fluorescence imaging}

Metastatic growth of disseminated breast cancer cells was followed by fluorescence imaging using the LT 9 GFP imaging system LT-MACIMSYSPLUSC (Lightools Research, Encinitas, CA, USA) on days 10, 14 (randomization), and after sacrifice. Autofluorescence was reduced using the chlorophyll-free diet. The animals were anesthetized and imaged in both the ventral and dorsal position. The imaging settings (exposure $2.34 \mathrm{~s}$, gain 6.7 and offset 238) were the same for all animals. The area of fluorescence was determined from

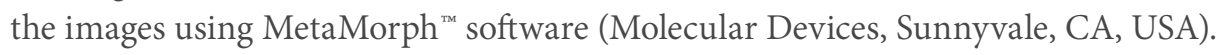

\section{Radiography}

Development of bone lesions was monitored by radiography on days 10, 14 (randomization), and prior to sacrifice. The animals were anesthetized and radiographed ( $31 \mathrm{kV}, 10 \mathrm{~s}, 2 \times$ magnification) in a prone position using the faxitron specimen radiographic system MX-20 DC-2 and Specimen Radiography Beta 2.0.0 software (both from Faxitron Bioptics, LLC, Tucson, AZ, USA). Lesion number and area were determined in tibias and femurs and the resulting data were analyzed using MetaMorph ${ }^{\mathrm{Tw}}$ software. 


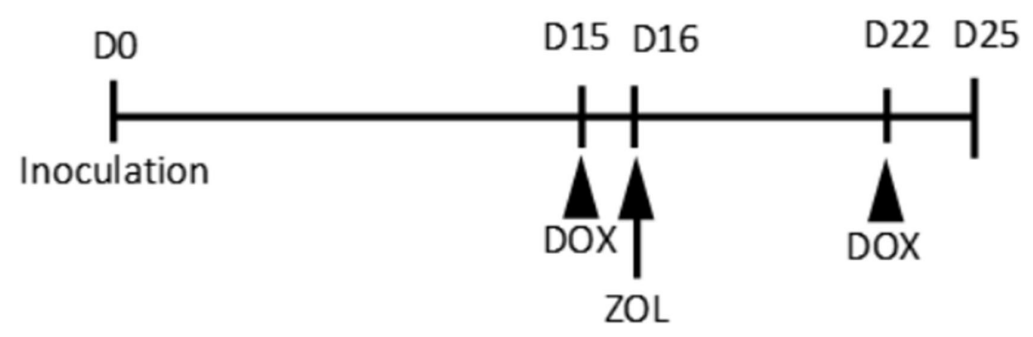

Figure 1. Dosing schedule of the doxorubicin (DOX) + zoledronic acid (ZOL) group

\section{Histomorphometric analyses}

After fixation in 10\% neutral buffered formalin and decalcification in $10 \%$ ethylenediaminetetraacetic acid for two weeks, the bone samples were processed to paraffin blocks. Four $\mu \mathrm{m}$ thick midsagittal sections were obtained from the left hind limb (tibia and femur) and stained with hematoxylin, eosin, Orange G and phloxine $\mathrm{B}(\mathrm{HE}+\text { Orange } \mathrm{G})^{[16]}$, tartrate-resistant acid phosphatase (TRAP) ${ }^{[6]}$, Ki-67 (primary antibody clone SP6, Nordic BioSite AB, Täby, Sweden) and terminal deoxynucleotidyl transferase dUTP nick end labeling (TUNEL) methods (in situ cell death detection kit, AP ${ }^{\mathrm{m}}$, Roche Diagnostics GmbH, Basel, Switzerland). Tumor area as well as trabecular and cortical bone areas were analyzed from the HE + Orange G stained slides as follows: first, micrographs were taken with a Leica DM4000 B Research Microscope (Leica Microsystems, Wetzlar, Germany), then brightness and contrast were optimized using the same settings for all images. Next, the tumor area was quantitated by drawing and bone area was quantitated by color tresholding using MetaMorph ${ }^{\mathrm{m}}$ software as described ${ }^{[8]}$. Areas expanding $5 \mathrm{~mm}$ from the articular surfaces of femur and tibia were analyzed. From a TRAP stained section, the number of osteoclasts at the tumor-bone interface in distal femur and proximal tibia was counted as described ${ }^{[18]}$, the whole section was analyzed using the MetaMorph ${ }^{\mathrm{m}}$ software. Apoptotic cells in tumor were manually counted as TUNEL stained cells with apoptotic morphology as described ${ }^{[6]}$. The whole tumor area was analyzed using a $20 \times$ objective Leica DM4000 B Research Microscope.

\section{Biomarker analyses}

$\mathrm{Ca}^{2+}$ concentration (corrected to $\mathrm{pH} 7.4$ by the internal algorithm of the instrument) in whole blood was determined using a blood gas analyzer (ABL835 Flex blood gas analyzer, Radiometer Medical ApS, Bronshoj, Denmark) immediately after terminal bleeding. A comparison to the normal level of ionized calcium was performed using the values obtained from five intact animals of the same strain, sex and age. Serum tartrateresistant acid phosphatase $5 \mathrm{~b}$ (TRACP 5b) activity as a marker of osteoclast number was measured in serum samples obtained on days 1, 9, 17 and 24 using the MouseTRAP kit (IDS, Boldon, UK).

\section{Statistical analyses}

Statistical analysis was performed with SPSS (version 14.0). All statistical analyses were performed as twosided tests and $P<0.05$ was considered statistically significant. The normality of residuals and homogeneity of variances were examined using Kolmogorov-Smirnov and Levene's tests, respectively. If these assumptions were fulfilled as such or after appropriate transformation, one-way ANOVA followed by Dunnett $t$-test was used. If the assumptions were not fulfilled even after transformation, the non-parametric Kruskal-Wallis test followed by Mann-Whitney $U$-test was used. Fischer Exact test was used for comparison of proportions.

\section{RESULTS}

\section{Effects of DOX and ZOL on body weight and tumor characteristics}

Body weight of the mice decreased during the last five days of the study due to the disease progression. Although the weight loss was more pronounced in the DOX treated groups, statistically significant differences compared to vehicle group in the body weight change from day 0 were not observed [Figure $2 \mathrm{~B}$ ]. 


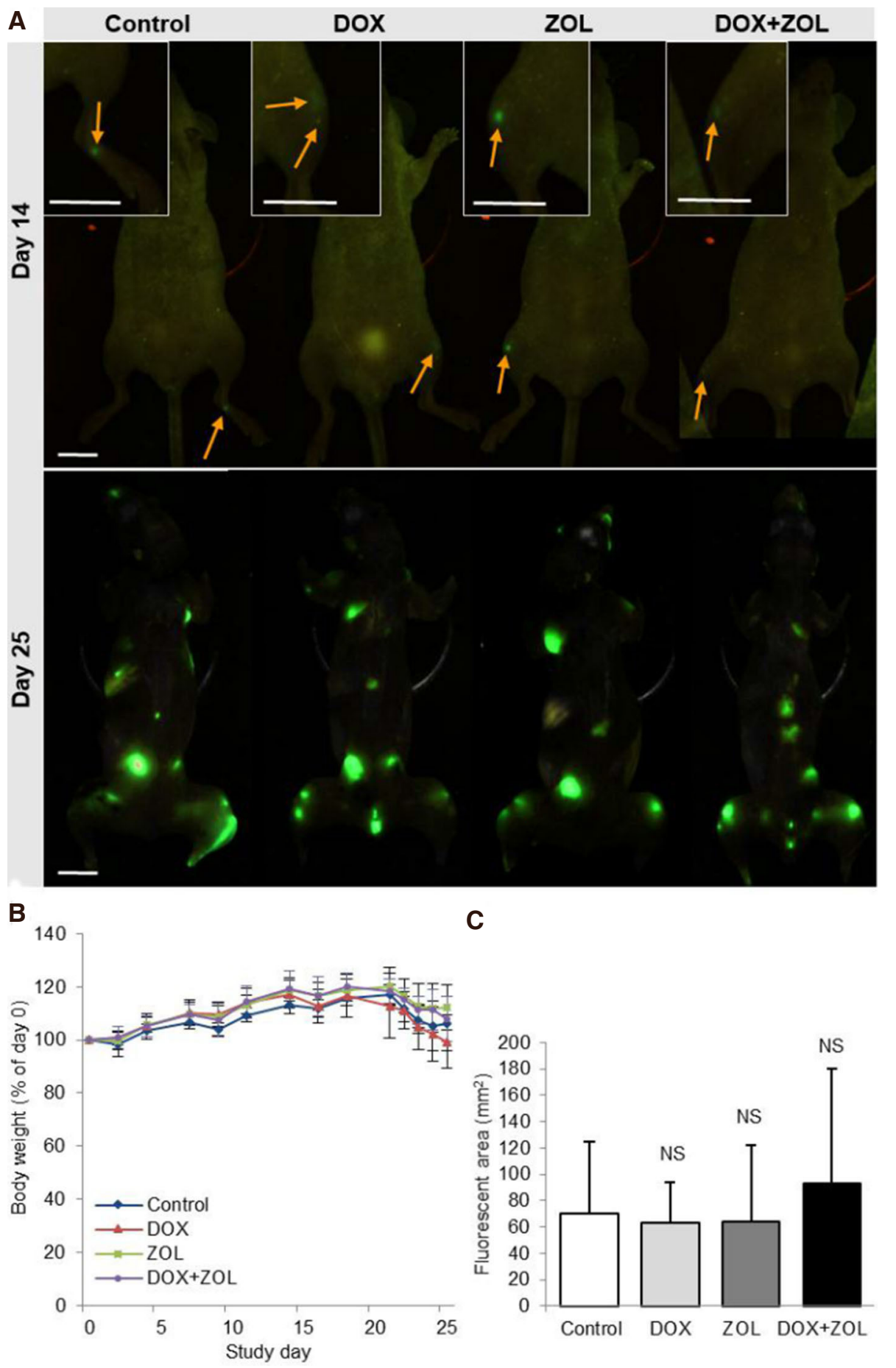

Figure 2. Body weight and MDA-MB231(SA) tumor burden. Whole body tumor burden was analyzed by fluorescence imaging. A: Representative images showing established metastatic foci before treatment (day 14) and at sacrifice (day 25); B: body weight during the study relative to body weight at the day of tumor cell inoculation. Significant differences in body weight at sacrifice relative to baseline were not observed; $\mathrm{C}$ : whole body tumor burden did not differ between the groups as measured by fluorescent imaging. Data are expressed as mean $\pm S D, n=8$ animals. Scale bars $10 \mathrm{~mm}$, arrows tumor foci in vivo. NS: non-significant; DOX: doxorubicin; ZOL: zoledronic acid

Anti-tumor effects of DOX, ZOL, and their sequential combination were studied by (1) measuring whole body tumor burden by fluorescence imaging; and (2) tumor area in bone by histomorphometry [Figures $2 \mathrm{C}$ and $3 \mathrm{D}$, respectively]. According to fluorescence imaging at sacrifice, none of the treatments affected the 
Table 1. The number of bone and soft tissue metastases were counted from green fluorescent protein images taken from dorsal and ventral sides of each animal

\begin{tabular}{|c|c|c|c|c|c|}
\hline Groups & & Vehicle & DOX & ZOL & $\mathrm{DOX}+\mathrm{ZOL}$ \\
\hline \multirow{3}{*}{ Bone } & Mean & 6.63 & 6.75 & 5.87 & 7.0 \\
\hline & SD & 2.33 & 2.38 & 3.91 & 4.0 \\
\hline & ANOVA & \multicolumn{4}{|c|}{$P=0.913$} \\
\hline \multirow{3}{*}{ Soft tissue } & Mean & 0.50 & 0.75 & 0.50 & 0.43 \\
\hline & SD & 0.76 & 1.03 & 0.76 & 0.79 \\
\hline & ANOVA & \multicolumn{4}{|c|}{$P=0.884$} \\
\hline
\end{tabular}

DOX: doxorubicin; ZOL: zoledronic acid

whole body tumor burden [Figure $2 \mathrm{C}$ ]. Furthermore, there was no difference in the numbers of metastases to bone or soft tissue sites between the treatment groups, based on fluorescence imaging [Table 1].

In accordance to these results, none of the treatments decreased the tumor area in bone analyzed from histological sections [Figure 3D]. All sections except sections from one animal in the DOX + ZOL group, contained tumor tissue. In many sections the tumor filled the entire intraosseous area. A thin layer of tumor on the periosteal surface resulting from disruption of the cortical envelope was sometimes observed. The anti-tumor effects were further examined by counting the number of apoptotic tumor cells and the proliferation index in histological samples [Figure $3 \mathrm{E}$ and $\mathrm{F}$ ]. Even though the intraosseus tumor area was not affected, the number of apoptotic cancer cells analyzed by TUNEL staining was increased in the DOX and DOX + ZOL groups [Figure 3E]. However, the sequential combination of DOX and ZOL did not increase the amount of apoptotic cells compared to DOX alone.

\section{Effects of DOX and ZOL on bone}

The effects of DOX and ZOL on bone were studied by measuring osteolytic lesion areas observed in X-ray images and trabecular bone area in histological sections. All mice in the control and DOX groups, $12.5 \%$ of the mice in the ZOL group, and $42.9 \%$ of the mice in the DOX + ZOL group had osteolytic lesions at sacrifice [Figure 4A]. The proportion of mice with osteolytic lesions was significantly lower in both groups treated with ZOL compared to the control group $(P<0.001$ for ZOL and $P=0.026$ for DOX $+\mathrm{ZOL}$ ). Osteolytic lesion area measured from X-ray images was radically lower in the ZOL and DOX + ZOL groups compared to the control group [Figure $4 \mathrm{~B}$ ]. ZOL also increased trabecular bone area by a factor of 2 analyzed by histomorphometry and DOX + ZOL by a factor of 1.5 compared to control. However, in the DOX + ZOL group statistically significant difference to the control was not reached [Figure $4 \mathrm{C}$ ].

\section{Effects of DOX and ZOL on TRACP $5 b$ and $\mathrm{Ca}^{2+}$ concentration in the blood}

Because breast cancer patients with bone metastases often have hypercalcemia and increased serum TRACP $5 \mathrm{~b}$ has also consistently been reported in these patients, we measured $\mathrm{Ca}^{2+}$ in blood and serum TRACP 5b from the mice. In addition, osteoclasts were counted at tumor-bone interface of TRAP-stained sections. We found that ZOL alone rapidly decreased serum TRACP 5b values as early as two days after dosing, on day 17. Both the ZOL and DOX + ZOL groups showed decreased TRACP $5 \mathrm{~b}$ on day 24 compared to the control group [Figure $5 \mathrm{~B}$ ]. The number of osteoclasts was $25 \%-50 \%$ lower at the tumor-bone interface in the ZOL and DOX + ZOL treated groups compared to the vehicle group [Figure $5 \mathrm{C}$ ], but the differences were not statistically significant $\left(P=0.595\right.$ and 0.142 , respectively). Both groups also had lower blood $\mathrm{Ca}^{2+}$ compared to the control group (5d). The blood $\mathrm{Ca}^{2+}$ in ZOL and DOX + ZOL groups was similar to the baseline $\mathrm{Ca}^{2+}$ measured from five healthy animals of same age, sex and strain $(1.268 \mathrm{mmol} / \mathrm{L}, \mathrm{SD} 0.0205)$, whereas the control group had higher blood $\mathrm{Ca}^{2+}$ compared to the healthy animals $(P=0.038)$. In the DOX group $4 / 8$ animals were above the range of healthy mice $(>1.29 \mathrm{mmol} / \mathrm{L})$, but the group average did not differ from the control group. 

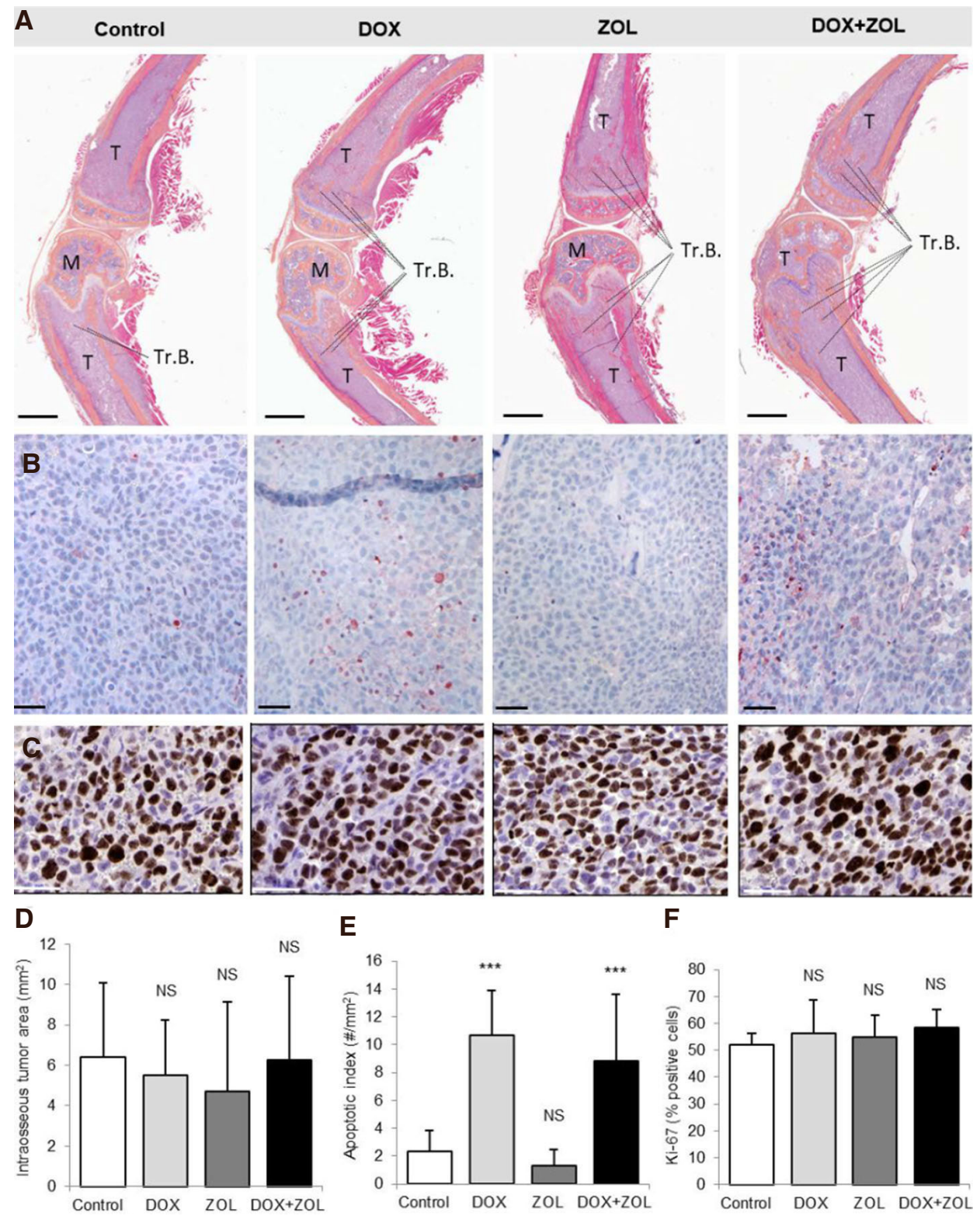

Figure 3. Intraosseous tumor area, apoptosis and proliferation. Tumor burden in bone was analyzed in histological sections of the left hind leg. Apoptotic tumor cells were stained using the TUNEL method and stained cells with apoptotic morphology were counted. Proliferation index was analyzed from Ki-67 stained sections. A: Representative images of HE + Orange G stained sections showing tumors in bone at sacrifice (day 25); B: representative images of the TUNEL stained sections; C: representative images of the Ki-67 stained sections; D: intraosseus tumor area did not differ between the groups; E: apoptotic tumor cells relative to tumor area were increased in both groups receiving DOX, but not in the ZOL group; F: proliferation index did not differ between the groups. Data are expressed as mean $\pm \mathrm{SD}, n=$ 8 animals. Scale bars $1 \mathrm{~mm}(A) ; 50 \mu \mathrm{m}$ (B and C). ${ }^{\star \star \star}$ Significantly different from control group $(P<0.001)$; NS: non-significant; T: tumor; M: marrow; Tr.B.: trabecular bone; HE: hematoxylin-eosin; TUNEL: terminal deoxynucleoitidyl transferase dUTP nick end labeling; DOX: doxorubicin; ZOL: zoledronic acid

\section{DISCUSSION}

Direct anti-tumor effects of bisphosphonates on established tumors in bone have been reported in many preclinical studies ${ }^{[5-7]}$. Decrease in tumor growth has been observed also in a model with defective osteoclasts ${ }^{[19]}$. However, findings have not been positive in all models, and clear clinical proof has been lacking. Additive or synergistic effects when combined with chemotherapeutic agents observed in some preclinical models of bone metastases as well as primary tumors have further fueled the interest in the anti-tumor actions of bisphosphonates. Several clinical studies with combination of neoadjuvant 
A
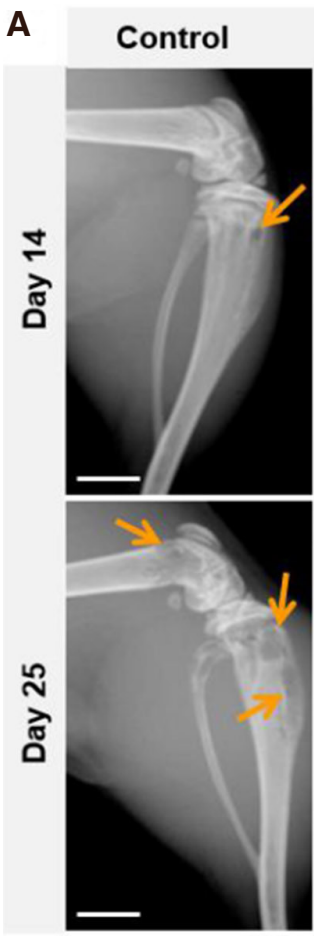

B

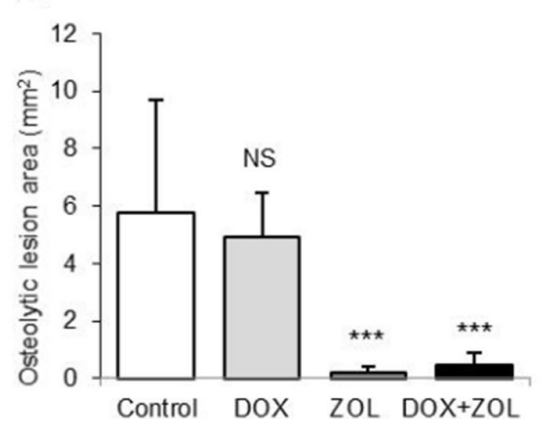

DOX
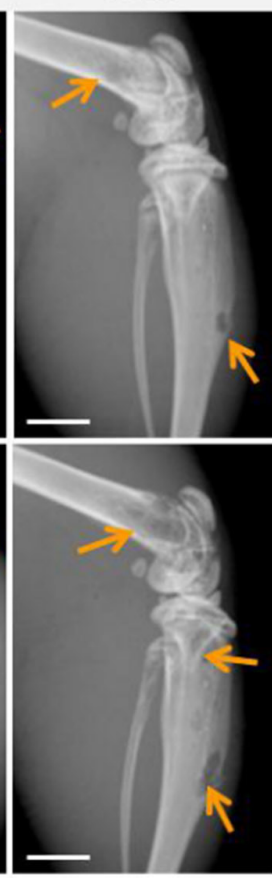

C
ZOL
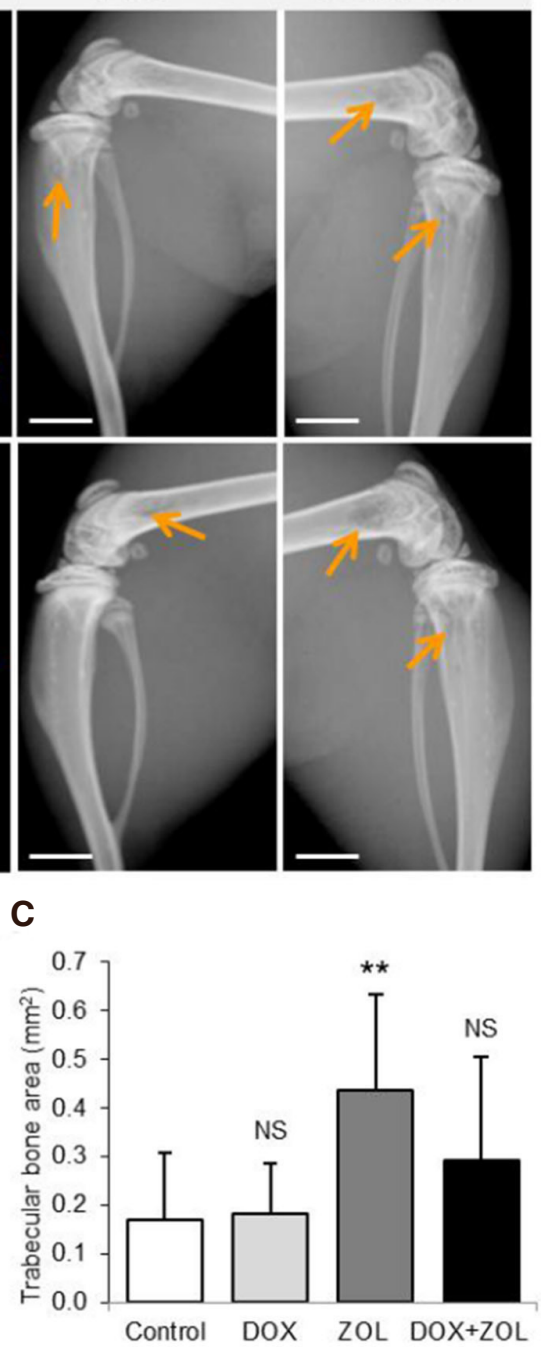

Figure 4. Osteolytic lesions, trabecular bone volume and the number of osteoclasts at the bone-tumor interface. Osteolytic lesion area was analyzed from X-ray images of both hind limbs, trabecular bone area and the number of osteoclasts in the bone-tumor interface in midsagittal histological sections of the left hind limb. A: Representative images showing established osteolytic lesions before treatment and at sacrifice; B: osteolytic lesion area was decreased in the ZOL and DOX + ZOL groups as measured by radiography; C: trabecular bone area was increased in the group receiving only ZOL, but the effect was not statistically significant in the DOX $+Z O L$ group. Data are expressed as mean $\pm \mathrm{SD}, n=8$ animals. Scale bars $2 \mathrm{~mm}$, arrows osteolytic lesions. ${ }^{\star \star}$ Significantly different from control group ( $\left.P<0.01\right)$; ${ }^{\star \star \star}$ significantly different from control group $(P<0.001)$; NS: non-significant; DOX: doxorubicin; ZOL: zoledronic acid

chemotherapeutic agent and ZOL have been reported. A retrospective analysis of sequential treatment showed benefit in a patient subgroup of postmenopausal women with estrogen receptor negative breast cancer ${ }^{[20]}$. Subsequent randomized controlled trials have suggested benefit, but failed to show statistically significant results ${ }^{[2-23]}$. This study aimed to examine whether a sequential combination of DOX and single dose ZOL has synergistic effects in the widely used MDA-MB-231(SA) bone metastasis model.

Aminobisphosphonates, such as ZOL, inhibit the mevalonate pathway and thus protein prenylation, resulting in inhibition of many subsequent cellular processes and induction of apoptosis in in vitro studies, but the results of in vivo studies are less conclusive ${ }^{[24]}$. ZOL alone did not affect tumor burden, proliferation or apoptosis, which is in line with previous findings, including models using MDA-MB231 cells or its sublines $s^{[5,8,1,1,2]}$. It is also in line with clinical data of ZOL not increasing overall survival in patients with 

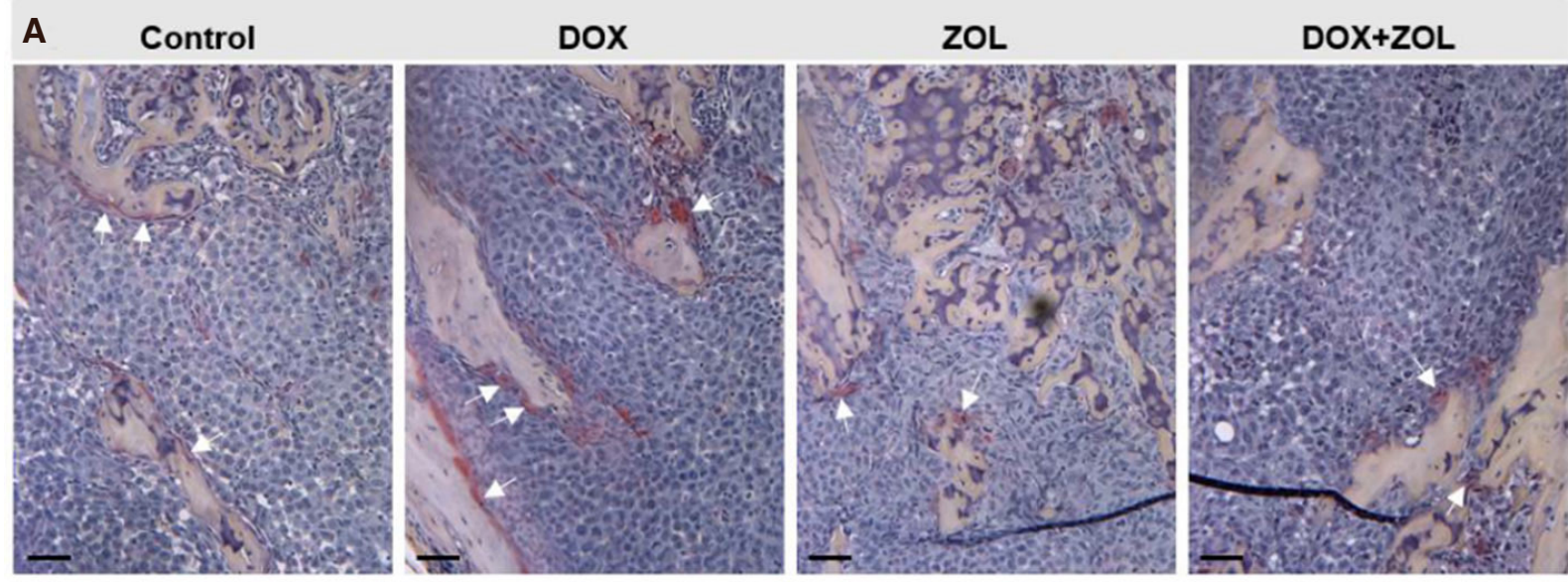

B

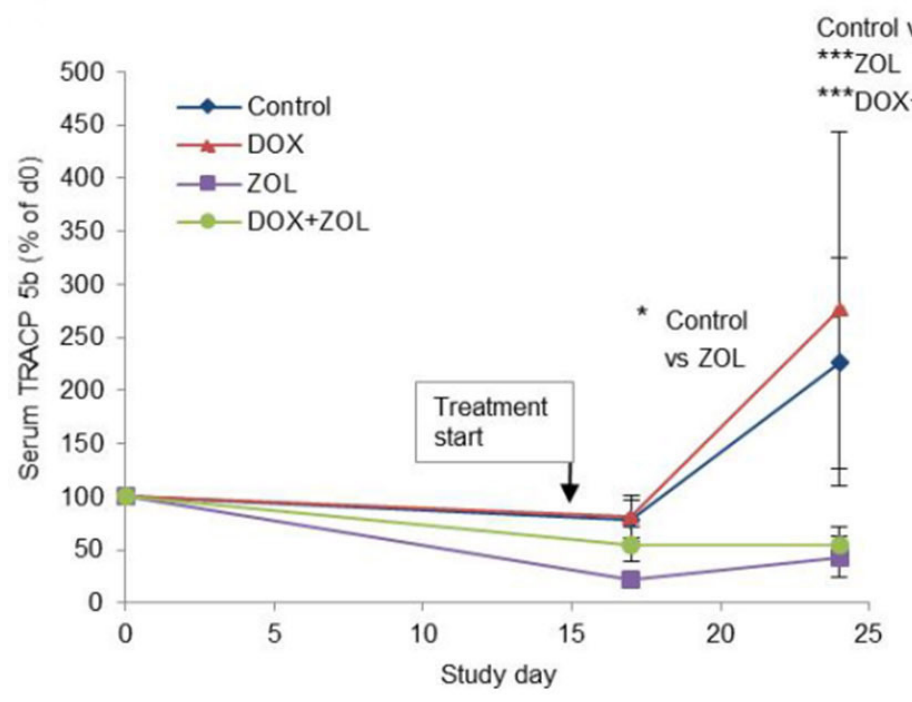

\section{C}

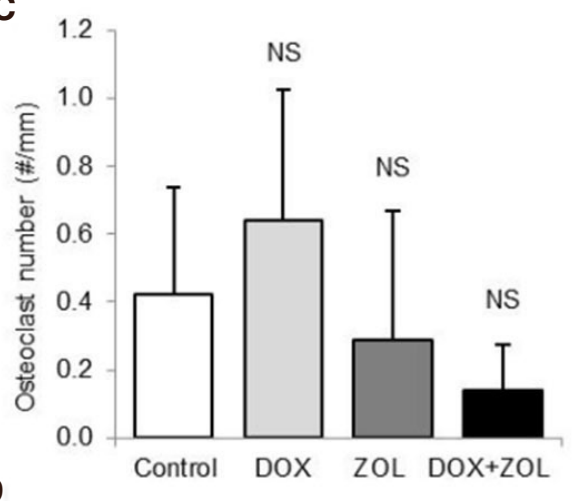

D

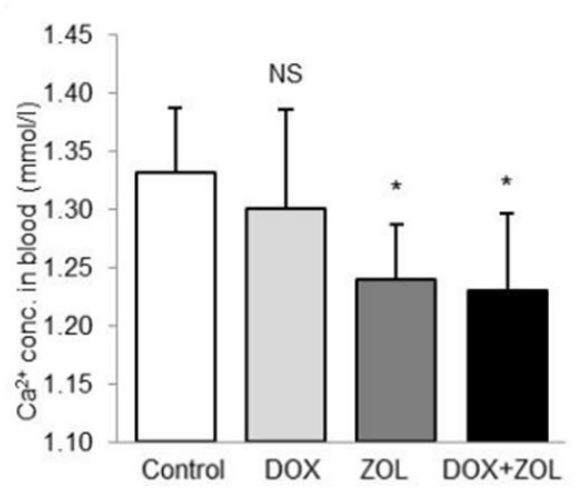

Figure 5. Osteoclasts and blood $\mathrm{Ca}^{2+}$. Osteoclasts were counted from TRAP stained sections and serum TRACP $5 \mathrm{~b}$ activity was measured at three time points (before inoculation of cells, days 17 and 24) as a systemic marker of osteoclast number. Blood $\mathrm{Ca}^{2+}$ was measured from terminal blood samples for detection of hypercalcemia. A: Representative images of the TRAP staining; B: statistically significant changes were not observed in the number of osteoclasts at tumor-bone interface; C: serum TRACP $5 \mathrm{~b}$ was decreased in the ZOL group already on day 17 and in the ZOL and DOX + ZOL groups on day 24 compared to the control group; D: hypercalcemia was prevented in the ZOL and DOX + ZOL groups. Data are expressed as mean $\pm \mathrm{SD}, n=7-8$ animals. Scale bars $50 \mu \mathrm{m}$, arrows osteoclasts. ${ }^{\star}$ Significantly different from control group $(P<0.05)$; ${ }^{\star \star \star}$ significantly different from control group $(P<0.001)$; NS: non-significant; TRAP: tartrate resistant acid phosphatase; TRACP 5b: tartrate resistant acid phosphatase 5b; DOX: doxorubicin; ZOL: zoledronic acid

established bone metastases ${ }^{[3]}$. DOX on the other hand did increase the number of apoptotic cells, as expected. We did not observe any other significant effects with DOX on tumor growth or proliferation index, which may have been due to a suboptimal dose of DOX. Higher doses of DOX are able to decrease tumor burden in the same model ${ }^{[25]}$. However, the dose in this study was set at a low level to enable observation of possible synergistic effects. Likewise, Ottewell et al ${ }^{[11]}$ used a suboptimal dose of DOX and observed synergistic effects on tumor volume and number of apoptotic cells in bone. They did not observe any effects of DOX alone on apoptosis by caspase-3 staining or on tumor burden in the intravenous MDA- 
$\mathrm{MB} 231 \mathrm{BO} 2$ or intracardiac MDA-MB231 models. In contrast, we observed clear induction of apoptosis with DOX alone, but we did not observe any potentiation of apoptosis induction with the sequential combination. Furthermore, the sequential DOX + ZOL treatment did not decrease tumor area in our study, despite of increased apoptosis of cancer cells induced by DOX. The absence of any observed decrease in the GFP positive area representing total tumor burden supports the histological data.

The metastatic behavior between cell sublines can be strikingly different. Sublines of the MDA-MB231 cells derived through in vivo selection for bone metastases behaved differently, some formed metastases in adrenal glands and others in bone, some were fast with a high frequency and others were slow with a low frequency of metastases ${ }^{[26]}$. However, the bone or adrenal metastatic sublines of the MDA-MB231 had similar growth rate when inoculated subcutaneously demonstrating that the cells were not generally more aggressive. Importantly, the bone metastatic subline cells exclusively induced osteoclast mobilization and recruitment ${ }^{[26]}$. The MDA-MB231(SA) model is more aggressive and progresses faster than the MDA-MB231BO2 model, as the median survival time is 25 days in the former ${ }^{[8]}$ and 60 days in the latter ${ }^{[11]}$, with the same number of inoculated cells. However, the different inoculation route is likely to play a role in the disease progression. It is possible that more cells are seeded to the bone marrow through the intracardiac route, than through the intravenous route, where the cells have to pass through the lung capillaries first. The MDA-MB231(SA) cells have been found to express, e.g., more serine and interleukin-11 compared to the parental MDA-MB231 cells ${ }^{[27,28]}$, whereas the MDA-MB231BO2 cells were found to have decreased expression of the miRNA30 family regulating several osteomimicry genes, compared to the parental cells ${ }^{[29]}$. Although the history of the two sublines is very different, the $\mathrm{BO}_{2}$ subline being derived through in vivo selection and the cells from San Antonio having occurred spontaneously, a common finding for both MDA-MB231(SA) and -BO2 sublines compared to the parental line is the strong downregulation of miRNAs 200a and $429^{[28,29]}$. These miRNAs are involved in the epithelial-to-mesenchymal transition ${ }^{[30]}$. Positive effects on tumor growth and survival have also been observed in a model of MDA-MB436 intratibial tumors using intensive, weekly sequential administration of DOX $+\mathrm{ZOL}^{[14]}$. The MDA-MB436 cells are triple negative like MDA-MB231 cells, and both represent the claudin-low subtype.

As expected, ZOL efficiently prevented the progression of osteolytic lesions as both monotherapy and in combination with DOX. However, trabecular bone area was not statistically significantly higher in the DOX + ZOL group compared to the control group. The lesions visible in X-ray images present mainly lesions in cortical bone, whereas the reduction of trabecular bone is typically not so clearly observed in the X-ray images. This could partly explain the difference in radiography and histological data. Furthermore, a lesion has lost $50 \%$ of the bone volume by the time it is visible with planar X-ray imaging. MicroCT analysis would provide volumetric information of both cortical and trabecular compartments. Unfortunately, this imaging modality was not available for this study. Furthermore, there were no statistically significant differences in the numbers of osteoclasts at the tumor-bone interface between the groups. The variation of osteoclast number measurement was high, affected by the variable amount of measurable tumor-bone interface in the samples. The reason for not observing significant reduction in numbers of osteoclasts at the tumorbone interface might be that the ZOL-induced reduction in the number of osteoclasts has been reported to be transient, observed three days after ZOL dosing to mice, but non-observable already five days after ZOL administration due to the formation of new osteoclasts ${ }^{[31]}$. Consistently, serum TRACP $5 b$ was decreased three days post-dosing compared to the control group. Serum TRACP $5 \mathrm{~b}$ remained low at later time points in the groups treated with ZOL, suggesting that the osteoclasts may have been smaller ${ }^{[32]}$, or that the number of osteoclasts along normal bone surface at tumor-free areas was lower. Indeed, Brown et al. ${ }^{[12]}$ have reported decreased number of osteoclasts in tumor-free bone but not in tumor-bearing bone 13 days after administration of ZOL. Very importantly, however, the function of the osteoclasts at the tumor-bone interface was inhibited, as shown by the decreased osteolytic lesion area in the ZOL and DOX + ZOL groups, the increased trabecular bone area in the ZOL group, and the prevention of hypercalcemia in the ZOL and $\mathrm{DOX}+\mathrm{ZOL}$ groups. 
High variation was a problem in this study and may have masked treatment effects on osteoclast number. Larger groups and more sensitive methods, such as $\mu \mathrm{CT}$ and luciferase labelled cells could have helped in reducing the variation. Intraosseus tumor area can only be analyzed by histology, which was restricted to analyzing the midsagittal sections in order to standardize the analysis. However, analyzing several levels could have reduced the variation. In order to further clarify the reasons underlying the insensitivity of the MDA-MB231(SA) tumors in bone to the combination treatment, as well as the unresponsive portion of patient population, the ZOL uptake of the MDA-MB231(SA) cells after DOX administration requires investigation. Longer treatment schedules with repeated cycles of sequential combinations would better present the clinical situation, but such studies are unfortunately not possible in this model due to the time frame of disease progression. Furthermore, the strong bone forming ability of the young, fast growing mice used in this model presents a major difference to the elderly breast cancer patients. New bone formed after the $\mathrm{ZOL}$ administration is not protected by $\mathrm{ZOL}$, and is thus vulnerable to cancer induced degradation. This could have mitigated the effects of ZOL in this study, but because the treatment period is so short, the amount of new bone formed in that time period is not likely to influence the results.

In summary, we examined the anti-tumor effects of a single dose of ZOL alone and in sequential combination with DOX on established bone metastases in the intracardiac MDA-MB231(SA) model. DOX induced apoptosis in the tumor cells and ZOL prevented tumor-induced bone destruction. However, antitumor effects of ZOL were not observed, nor additive or synergistic effects of the sequential combination of DOX and ZOL.

\section{DECLARATIONS}

\section{Acknowledgments}

We gratefully acknowledge Nanna Merikoski, Johanna Örling and Suvi Suutari for their skillful technical assistance and Aurexel Life Sciences Ltd. for the editorial support.

\section{Authors' contributions}

Conception and design of the study, data analysis and interpretation: Suominen MI

Data acquisition, provided administrative, technical, and material support: Rissanen JP, Käkönen R, Halleen JM

Revised the manuscript: Härkönen P, Käkönen SM

\section{Availability of data and materials}

The data used to support the findings of this study are available from the corresponding author upon request.

\section{Financial support and sponsorship}

None.

\section{Conflicts of interest}

Suominen MI, Rissanen JP, Halleen JM and Härkönen P are voting stock holders of Pharmatest Services Ltd. Käkönen SM and Käkönen R are voting stock holders of Aurexel Life Sciences. Halleen JM is a consultant of and receipt of royalties from IDS Plc.

\section{Ethical approval and consent to participate}

All applicable international, national, and/or institutional guidelines for the care and use of animals were followed. 


\section{Consent for publication}

Not applicable.

\section{Copyright}

(c) The Author(s) 2019.

\section{REFERENCES}

1. Wilson C, Coleman R. Adjuvant bone-targeted therapies for postmenopausal breast cancer. JAMA Oncol 2016;2:423-4.

2. Body JJ, Terpos E, Tombal B, Hadji P, Arif A, et al. Bone health in the elderly cancer patient: a SIOG position paper. Cancer Treat Rev 2016;51:46-53.

3. Wong M, Pavlakis N. Optimal management of bone metastases in breast cancer patients. Breast Cancer (Dove Med Press) 2011;3:35-60.

4. Coleman R, Cameron D, Dodwell D, Bell R, Wilson C, et al. Adjuvant zoledronic acid in patients with early breast cancer: final efficacy analysis of the AZURE (BIG 01/04) randomised open-label phase 3 trial. Lancet Oncol 2014;15:997-1006.

5. Daubiné F, Le Gall C, Gasser J, Green J, Clézardin P. Antitumor effects of clinical dosing regimens of bisphosphonates in experimental breast cancer bone metastasis. J Natl Cancer Inst 2007;99:322-30.

6. Hiraga T, Williams PJ, Mundy GR, Yoneda T. The bisphosphonate ibandronate promotes apoptosis in MDA-MB-231 human breast cancer cells in bone metastases. Cancer Res 2001;61:4418-24.

7. Zekri J, Mansour M, Karim SM. The anti-tumour effects of zoledronic acid. J Bone Oncol 2014;3:25-35.

8. Suominen MI, Rissanen JP, Käkönen R, Fagerlund KM, Alhoniemi E, et al. Survival benefit with radium-223 dichloride in a mouse model of breast cancer bone metastasis. J Natl Cancer Inst 2013;105:908-16.

9. Junankar S, Shay G, Jurczyluk J, Ali N, Down J, et al. Real-time intravital imaging establishes tumor-associated macrophages as the extraskeletal target of bisphosphonate action in cancer. Cancer Discov 2015;5:35-42.

10. Rogers TL, Wind N, Hughes R, Nutter F, Brown HK, et al. Macrophages as potential targets for zoledronic acid outside the skeletonevidence from in vitro and in vivo models. Cell Oncol (Dordr) 2013;36:505-14.

11. Ottewell PD, Deux B, Mönkkönen H, Cross S, Coleman RE, et al. Differential effect of doxorubicin and zoledronic acid on intraosseous versus extraosseous breast tumor growth in vivo. Clin Cancer Res 2008;14:4658-66.

12. Brown HK, Ottewell PD, Evans CA, Coleman RE, Holen I. A single administration of combination therapy inhibits breast tumour progression in bone and modifies both osteoblasts and osteoclasts. J Bone Oncol 2012;1:47-56.

13. Ottewell PD, Mönkkönen H, Jones M, Lefley DV, Coleman RE, et al. Antitumor effects of doxorubicin followed by zoledronic acid in a mouse model of breast cancer. J Natl Cancer Inst 2008;100:1167-78.

14. Ottewell PD, Woodward JK, Lefley DV, Evans CA, Coleman RE, et al. Anticancer mechanisms of doxorubicin and zoledronic acid in breast cancer tumor growth in bone. Mol Cancer Ther 2009;8:2821-32.

15. Ottewell PD, Lefley DV, Cross SS, Evans CA, Coleman RE, et al. Sustained inhibition of tumor growth and prolonged survival following sequential administration of doxorubicin and zoledronic acid in a breast cancer model. Int J Cancer 2010;126:522-32.

16. Guise TA, Yin JJ, Taylor SD, Kumagai Y, Dallas M, et al. Evidence for a causal role of parathyroid hormone-related protein in the pathogenesis of human breast cancer-mediated osteolysis. J Clin Invest 1996;98:1544-9.

17. Arguello F, Frantz CN, Baggs RB. Experimental bone and bone marrow metastasis in laboratory animals. J Natl Cancer Inst 1990;82:106970.

18. Sasaki A, Boyce BF, Story B, Wright KR, Chapman M, et al. Bisphosphonate risedronate reduces metastatic human breast cancer burden in bone in nude mice. Cancer Res 1995;55:3551-7.

19. Hirbe AC, Roelofs AJ, Floyd DH, Deng H, Becker SN, et al. The bisphosphonate zoledronic acid decreases tumor growth in bone in mice with defective osteoclasts. Bone 2009;44:908-16.

20. Coleman RE, Winter MC, Cameron D, Bell R, Dodwell D, et al. The effects of adding zoledronic acid to neoadjuvant chemotherapy on tumour response: exploratory evidence for direct anti-tumour activity in breast cancer. Br J Cancer 2010;102:1099-105.

21. Horiguchi J, Hasegawa Y, Miura D, Ishikawa T, Hayashi M, et al. A randomized controlled trial comparing zoledronic acid plus chemotherapy with chemotherapy alone as a neoadjuvant treatment in patients with HER2-negative primary breast cancer. J Clin Oncol 2013;31:1029.

22. Fasching PA, Jud SM, Hauschild M, Kümmel S, Schütte M, et al. FemZone trial: a randomized phase II trial comparing neoadjuvant letrozole and zoledronic acid with letrozole in primary breast cancer patients. BMC Cancer 2014;14:66.

23. Charehbili A, van de Ven S, Smit VT, Meershoek-Klein Kranenbarg E, Hamdy NA, et al. Addition of zoledronic acid to neoadjuvant chemotherapy does not enhance tumor response in patients with HER2-negative stage II/III breast cancer: the NEOZOTAC trial (BOOG 2010-01). Ann Oncol 2014;25:998-1004.

24. Green JR. Antitumor effects of bisphosphonates. Cancer 2003;97:840-7.

25. Käkönen RS, Rissanen JP, Suominen MI, Halleen JM. Quantitative image analysis method for measuring whole-body tumor burden in a mouse model of breast cancer bone metastasis. J Bone Miner Res 2008;23:S136-243.

26. Kang Y, Siegel PM, Shu W, Drobnjak M, Kakonen SM, et al. A multigenic program mediating breast cancer metastasis to bone. Cancer Cell 2003;3:537-49.

27. Pollari S, Käkönen SM, Edgren H, Wolf M, Kohonen P, et al. Enhanced serine production by bone metastatic breast cancer cells stimulates osteoclastogenesis. Breast Cancer Res Treat 2011;125:421-30.

28. Pollari S, Leivonen SK, Perälä M, Fey V, Käkönen SM, et al. Identification of microRNAs inhibiting TGF- $\beta$-induced IL-11 production in bone metastatic breast cancer cells. PLoS One 2012;7:e37361. 
29. Heymann D. Bone cancer: primary bone cancers and bone metastases. 2th ed. Amsterdam: Elsevier/Academic Press; 2015. p. 486.

30. Gregory PA, Bert AG, Paterson EL, Barry SC, Tsykin A, et al. The miR-200 family and miR-205 regulate epithelial to mesenchymal transition by targeting ZEB1 and SIP1. Nat Cell Biol 2008;10:593-601.

31. Haider MT, Holen I, Dear TN, Hunter K, Brown HK. Modifying the osteoblastic niche with zoledronic acid in vivo-potential implications for breast cancer bone metastasis. Bone 2014;66:240-50.

32. Lv Y, Wang G, Xu W, Tao P, Lv X, et al. Tartrate-resistant acid phosphatase $5 \mathrm{~b}$ is a marker of osteoclast number and volume in RAW 264.7 cells treated with receptor-activated nuclear $\mathrm{\kappa B}$ ligand. Exp Ther Med 2015;9:143-6. 